\title{
BMJ Open The effect of luseogliflozin and alpha- glucosidase inhibitor on heart failure with preserved ejection fraction in diabetic patients: rationale and design of the MUSCAT-HF randomised controlled trial
}

\author{
Kentaro Ejiri, ${ }^{1}$ Toru Miyoshi, ${ }^{1}$ Kazufumi Nakamura, ${ }^{1}$ Satoru Sakuragi, ${ }^{2}$
} Mitsuru Munemasa, ${ }^{3}$ Seiji Namba, ${ }^{4}$ Atsushi Takaishi, ${ }^{5}$ Hiroshi Ito ${ }^{1}$

To cite: Ejiri K, Miyoshi T, Nakamura $\mathrm{K}$, et al. The effect of luseogliflozin and alphaglucosidase inhibitor on heart failure with preserved ejection fraction in diabetic patients: rationale and design of the MUSCAT-HF randomised controlled trial. BMJ Open 2019;9:e026590. doi:10.1136/ bmjopen-2018-026590

- Prepublication history and additional material for this paper are available online. To view these files, please visit the journal online (http://dx.doi org/10.1136/bmjopen-2018026590).

Received 10 September 2018 Revised 11 January 2019 Accepted 25 February 2019

Check for updates

(C) Author(s) (or their employer(s)) 2019. Re-use permitted under CC BY-NC. No commercial re-use. See rights and permissions. Published by BMJ.

For numbered affiliations see end of article.

Correspondence to

Dr Toru Miyoshi;

miyoshit@cc.okayama-u.ac.jp

\section{ABSTRACT}

Introduction Type 2 diabetes mellitus (T2DM) is a strong risk factor for coronary artery disease and heart failure, particularly heart failure with preserved ejection fraction (HFpEF). The aim of the ongoing MUSCAT-HF (It stands for Prospective Comparison of Luseogliflozin and Alphaglucosidase on the Management of Diabetic Patients with Chronic Heart Failure and Preserved Ejection Fraction) trial is to evaluate the efficacy of luseogliflozin, a sodiumglucose cotransporter 2 (SGLT2) inhibitor, versus voglibose, an alpha-glucosidase inhibitor, using brain natriuretic peptide (BNP) as the index of therapeutic effect in T2DM patients with HFpEF.

Methods and analysis A total of 190 patients with T2DM and HFpEF (ejection fraction $>45 \%$ ) who are drug-naïve or taking any anti-diabetic agents will be randomised (1:1) to receive luseogliflozin $2.5 \mathrm{mg}$ one time per day or voglibose $0.2 \mathrm{mg}$ three times per day. The patients will be stratified by age ( $<65$ years, $\geq 65$ years), baseline haemoglobin A1c $(<8.0 \%, \geq 8.0 \%)$, baseline BNP $(<100 \mathrm{pg} / \mathrm{mL}, \geq 100 \mathrm{pg} / \mathrm{mL})$, baseline renal function (estimated glomerular filtration rate $\geq 60 \mathrm{~mL} / \mathrm{min} / 1.73 \mathrm{~m}^{2},<60 \mathrm{~mL} / \mathrm{min} / 1.73 \mathrm{~m}^{2}$ ), use of thiazolidine or not and presence or absence of atrial fibrillation and flutter at screening. After randomisation, participants will receive the study drug for 12 weeks in addition to their background therapy. The primary endpoint is the proportional change in baseline BNP after 12 weeks of treatment. The key secondary endpoints are the change from baseline in the ratio of early mitral inflow velocity to mitral annular early diastolic velocity, body weight and glycaemic control after 12 weeks of treatment.

Ethics and dissemination The study has been approved by the ethics committee and the patients will be included after informed consent. The results will be submitted for publication in peer-reviewed journals.

Trial registration number UMIN000018395

\section{INTRODUCTION}

Type 2 diabetes mellitus (T2DM) is a strong risk factor for coronary artery disease and

\section{Strengths and limitations of this study}

- This study will be the first randomised controlled trial to evaluate the drug efficacy of an sodium-glucose cotransporter 2 inhibitor in the patients with type 2 diabetes mellitus and heart failure with preserved ejection fraction.

- This study is adequately powered to provide a clinically meaningful outcome.

- A 12 week intervention period may not be sufficient to see the full impact of treatment on long-term outcome.

heart failure, particularly, heart failure with preserved ejection fraction (HFpEF). ${ }^{1}$ A previous cohort study showed that the risk of heart failure was increased in the patients with T2DM. ${ }^{2}$ Therefore, the treatment of abnormal glucose metabolism is a promising strategy in the treatment of heart failure. However, large clinical trials have shown that intensive glucose-lowering treatment of hyperglycaemia, compared with less-intensive control treatment, did not decrease hospitalisation or mortality of heart failure. ${ }^{3}$ However, Kim et al reported that a alpha-glucosidase inhibitor regulated glucose metabolism and improved the pathophysiology of chronic heart failure in the patients with T2DM. ${ }^{4}$ The Study to Prevent Non-Insulin-Dependent Diabetes Mellitus (STOP-NIDDM) trial showed that the treatment of impaired glucose tolerance with a alpha-glucosidase inhibitor resulted in a significant reduction in the risk of cardiovascular disease. ${ }^{5}$ These data suggest that alpha-glucosidase inhibitors may be beneficial in the treatment of chronic heart failure. 
Recently, the Empagliflozin Cardiovascular Outcome Event Trial in Type 2 Diabetes Mellitus Patients-Removing Excess Glucose (EMPA-REG) OUTCOME ${ }^{67}$ and Canagliflozin Cardiovascular Assessment Study (CANVAS) Program $^{8} 9$ randomised controlled trials showed that sodium-glucose cotransporter 2 (SGLT2) inhibitors reduced all-cause mortality, cardiovascular mortality and hospitalisation of heart failure in T2DM compared with placebo. These results indicated that SGLT2 inhibitors may be effective in lowering glucose levels and reducing cardiovascular events, particularly in the patients with heart failure. Given that these trials were not specifically designed to investigate the effect of SGLT2 inhibitors in heart failure patients, no detailed data on their effects in heart failure were obtained.

The MUSCAT-HF (prospective comparison of luseogliflozin and alpha-glucosidase on the management of diabetic patients with chronic heart failure and preserved left-ventricular ejection fraction) trial described here is designed to evaluate the efficacy of luseogliflozin, an SGLT2 inhibitor, compared with voglibose, an alpha-glucosidase inhibitor, using brain natriuretic peptide (BNP) as the index of therapeutic effect in the patients with T2DM and HFpEF. The results of this study will support a novel strategy for the treatment of heart failure using an SGLT2 inhibitor, independent of its glucose-lowering effects.

\section{METHODS AND ANALYSIS \\ Study design}

The MUSCAT-HF trial is an ongoing, multi-centre, prospective, open-label, randomised controlled trial designed to assess the effect of luseogliflozin $(2.5 \mathrm{mg}$ one time per day) compared with voglibose $(0.2 \mathrm{mg}$ three times per day) on left ventricular load in the patients with T2DM and HFpEF. BNP level at 24 weeks after administration of the study drug will be used as a surrogate marker for heart failure.

\section{Study population}

The planned sample size of this study is 95 patients per group (190 patients in total). The recruitment of study patients is planned to take place from September 2015 to September 2018. The patients aged $\geq 20$ years with T2DM (haemoglobin A1c [HbA1C] $\leq 9.0 \%$ ) and HFpEF (left ventricular ejection fraction $\geq 45 \%$ ) needing additional treatment for T2DM despite the ongoing treatment are eligible for participation. The key inclusion and exclusion criteria are detailed in box 1. Given that the definition of chronic heart failure according to European Society of Cardiology guidelines includes $\mathrm{BNP} \geq 35 \mathrm{pg} / \mathrm{mL},{ }^{10}$ the patients with $\mathrm{BNP}<35 \mathrm{pg} / \mathrm{mL}$ will be excluded from this study. Study candidates will be assessed for eligibility within 4 weeks prior to enrolment (figure 1).

\section{Box 1 Detailed inclusion and exclusion criteria}

\section{Inclusion criteria}

1. Diagnosis of T2DM and left ventricular ejection fraction $>45 \%$ with current or previous symptoms of heart failure (dyspnoea on effort, orthopnoea or leg oedema)

2. Inadequately controlled T2DM in the patients who have received diet and exercise therapy, a lifestyle modification programme and hypoglycaemic medications based on standard guidelines of the Japan Diabetes Society

3. Age $>20$ years

4. Provision of written informed consent prior to participation

\section{Exclusion criteria}

1. $B N P<35 \mathrm{pg} / \mathrm{mL}$

2. Use of alpha-glucosidase inhibitors, SGLT2 inhibitors, glinides or high-dose sulfonylurea

3. Renal insufficiency (eGFR $<30 \mathrm{~mL} / \mathrm{min} / 1.73 \mathrm{~m}^{2}$ )

4. Left ventricular ejection fraction $<45 \%$

5. History of severe ketoacidosis or diabetic coma within 6 months prior to participation

6. Serious infection or severe trauma or perioperative patients

7. Type 1 diabetes mellitus

8. Poorly controlled T2DM (HbA1c $>9.0 \%)$

9. Uncontrolled hypertension (systolic blood pressure $>160 \mathrm{~mm} \mathrm{Hg}$ )

10. History of stroke, myocardial infarction or severe cardiovascular disease with hospitalisation within 6 months prior to participation

11. Women who are pregnant or breastfeeding

12. Allergy to either investigation product

13. Other medical reason at the investigator's discretion

BNP, brain natriuretic peptide; eGFR, estimated glomerular filtration rate; HbA1C, haemoglobin A1C; SGLT2, sodium-glucose cotransporter 2; T2DM, type 2 diabetes mellitus. BNP, brain natriuretic peptide; eGFR, estimated glomerular filtration rate; HbA1C, haemoglobin A1C; SGLT2, sodium-glucose cotransporter 2; T2DM, type 2 diabetes mellitus.

\section{Study outline and randomisation}

The patients fulfilling all criteria who provide written informed consent to participate in this study will be enrolled and subsequently randomised (1:1) to receive luseogliflozin (2.5 mg one time per day) or voglibose $(0.2 \mathrm{mg}$ three times per day) in addition to their background medication. Randomisation will be performed using a computer-generated random sequence web response system. The patients will be stratified by age ( $<65$ years, $\geq 65$ years), baseline HbA1c $(<8.0 \%, \geq 8.0 \%)$, baseline BNP $(<100 \mathrm{pg} / \mathrm{mL}, \geq 100 \mathrm{pg} / \mathrm{mL})$, baseline renal function (estimated glomerular filtration rate [eGFR] $\geq 60 \mathrm{~mL} / \mathrm{min} / 1.73 \mathrm{~m}^{2}, \quad<60 \mathrm{~mL} / \mathrm{min} / 1.73 \mathrm{~m}^{2}$ ), use of thiazolidine or not and presence or absence of atrial fibrillation (AF) and atrial fibrillation flutter (AFL) at screening.

Assessments during the study period are listed in figure 2. Laboratory data, ECG, echocardiography and the patients' vital signs, body weight and waist circumference, will be evaluated at $4 \pm 2$ weeks (visit $29 \pm 14$ days) and 12 weeks (visit $85 \pm 28$ days) after initiation of study treatment. Safety and tolerability will be assessed during the treatment period. The primary outcome of change in BNP compared with baseline will be evaluated at 12 weeks 

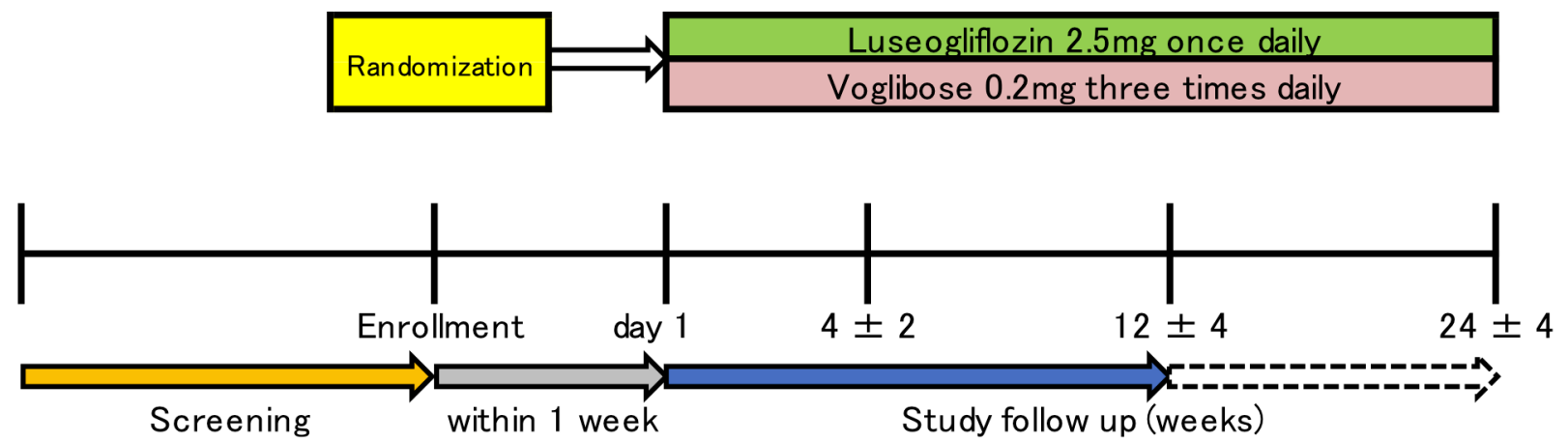

\section{(4 weeks)}

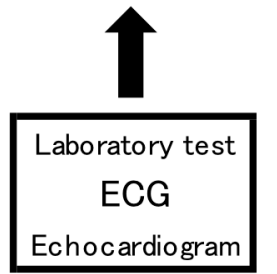

$*$ Base line

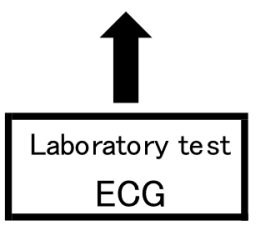

$E C G$

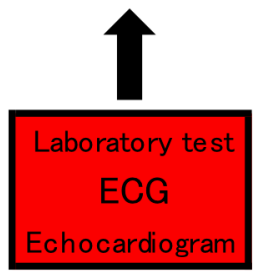

*Primary outcome

Figure 1 Study design. Arrows illustrate the patients' flow and the timing of follow-up. The patients with type 2 diabetes mellitus are screened whether with heart failure with preserved ejection fraction or without (screening period, yellow arrow). One of the study drugs was administered to the patients met inclusion criteria after collection of baseline data within 1 week after randomisation (grey arrow). After administration, mandatory follow-up period is for 12 weeks (study follow-up period, blue arrow). After 12 weeks, expanding follow-up are continued in the patients agreed with (arrow with dotted line). During expanding follow-up, the change of an allocated drug was not allowed. ECG, electrocardiogram.

(visit $85 \pm 28$ days) and the patient will be followed up for an additional 12 weeks (visit $169 \pm 28$ days) after the end of treatment. If a patient's glycaemic control worsens after $4 \pm 2$ weeks, the investigator can increase the dose of allocated treatment (to luseogliflozin $5 \mathrm{mg}$ one time per day or voglibose $0.3 \mathrm{mg}$ three times per day) and other specific T2DM drugs, except for sulfonylureas. Investigators will also be encouraged to treat all other cardiovascular risk factors according to the local standard of care. Under the following circumstances, the investigator must evaluate the data and the patient's vital sign: (1) discontinuation of study treatment, (2) dose increase of specific treatment for heart failure, (3) initiation of new treatment for heart failure and (4) withdrawal from the study. The permitted medications for the treatment of heart failure include angiotensin-converting enzyme inhibitors, angiotensin

\begin{tabular}{|c|c|c|c|c|}
\hline \multirow{2}{*}{ Assessment } & \multirow{2}{*}{ Enrollment } & \multicolumn{2}{|c|}{ Study follow-up period } & \multirow{2}{*}{$\begin{array}{c}\begin{array}{c}\text { Expanding follow-up } \\
\text { (if possible) }\end{array} \\
24 \text { weeks } \\
\text { (visit } 169 \pm 28 \text { days) }\end{array}$} \\
\hline & & $\begin{array}{c}4 \text { weeks } \\
\text { (visit } 29 \pm 14 \text { days) }\end{array}$ & $\begin{array}{c}12 \text { weeks } \\
\text { (visit } 85 \pm 28 \text { days) }\end{array}$ & \\
\hline Medical examination & $x$ & $x$ & $x$ & $x$ \\
\hline Written informed consent & $x$ & & & \\
\hline Clinical symptoms & $x$ & $x$ & $x$ & $x$ \\
\hline Adverse events & & $x$ & $x$ & $x$ \\
\hline Treatment discontinuation & & $x$ & $x$ & $x$ \\
\hline Vital signs & $x$ & $x$ & $x$ & $x$ \\
\hline Body weight & $x$ & $x$ & $x$ & $x$ \\
\hline Waist circumference & $x$ & & $x$ & $x$ \\
\hline Laboratory tests & $x$ & $x$ & $x$ & $x$ \\
\hline Electrocardiogram & $x$ & $x$ & $x$ & $x$ \\
\hline Echocardiography & $x$ & & $x$ & $x$ \\
\hline
\end{tabular}

Figure 2 Assessments during the study period. 
receptor blockers, beta-blockers, diuretics and mineralocorticoid/aldosterone receptor antagonists.

\section{Outcomes}

Primary outcome

The primary outcome of this study is the difference in BNP after 12 weeks (visit $85 \pm 28$ days) of treatment between the luseogliflozin and the voglibose groups, defined as the difference in logarithmic BNP change calculated as follows:

(*) BNP proportional change $=$ BNP (at follow-up) $/$ BNP (at baseline),

$(\dagger)$ Logarithmic BNP change $=$ logarithmic BNP (at follow-up) - logarithmic BNP (at baseline),

In other words, $(*)=$ exponential $(\dagger)$.

Furthermore, we calucurated the ratio of BNP change rate

$(\ddagger)$ The ratio of BNP proportional change (the luseogliflozin group to the voglibose group $)=(*)$ (in the luseogliflozin group)/(*) (in the voglibose group),

(§) The difference of logarithmic BNP change $=(\dagger)($ in the luseogliflozin group) $-(\dagger)$ (in the voglibose group),

In other words, $(+)=$ exponential $(\S)$

\section{Secondary outcomes}

The key secondary outcomes of this study are the differences in the following parameters between the luseogliflozin and the voglibose groups:

1. Ratio of early mitral inflow velocity to mitral annular early diastolic velocity (E/e'),

2. Left ventricular ejection fraction,

3. Body weight,

4. HbA1c.

The difference in $\mathrm{E} / \mathrm{e}^{\prime}$ and $\mathrm{HbA1c}$ between the groups is defined as the difference in logarithmic $\mathrm{E} / \mathrm{e}^{\prime}$ and HbAlc using the same calculation as for BNP. Difference in body weight and left ventricular ejection fraction is defined as the difference between those parameters at follow-up and at baseline. Further exploratory analysis is listed in online supplementary file 1.

\section{Safety outcomes: including, but not limited to}

- Clinical laboratory tests, vital signs, 12-lead ECG, physical examination and the use of rescue medication.

- Adverse events including major adverse cardiovascular events (MACE), hypoglycaemic adverse events (requiring any intervention) and urinary tract infection.

Safety will be assessed based on adverse events reported throughout the study, clinical laboratory tests, vital signs, 12-lead ECG, physical examination and the use of rescue medication. Prespecified adverse events include MACE, hypoglycaemic adverse events (requiring any intervention) and urinary tract infection (details listed in online supplementary file 1 ).

\section{Study oversight and organisation}

Members of the Steering Committee also designed the study and are responsible for its conduction (details listed in online supplementary file 2). Significant adverse events (SAEs) occurring within 30 days after final administration of the study drug or after 30 days with a suspicion of association with the study drug, as well as all pregnancies, will be immediately reported to the Steering Committee and the sponsor by the investigator, in accordance with good clinical practice.

\section{Statistical analysis}

Sample size and power calculation

The primary hypothesis of this study is that the SGLT2 inhibitor luseogliflozin can reduce cardiac load in the patients with T2DM and HFpEF. Therefore, the primary outcome was the difference in change in BNP from baseline to 12 weeks between the patients receiving luseogliflozin or voglibose. As of the start of recruitment in September 2015, no interventional study of the effect of SGLT2 inhibitors on heart failure in the patients with T2DM has been reported. Therefore, we estimated that BNP change rate in the luseogliflozin group will be $30 \%$ lower as compared with that in the voglibose group according to previous studies of the effect of renin-angiotensin-aldosterone system inhibitors on heart failure. ${ }^{11-13}$ The SD of the natural logarithmic transformation of BNP was estimated at 0.83 , in reference to the Prospective comparison of ARNI with ARB on Management Of heart failUre with preserved ejectioN fracTion (PARAMOUNT) study. ${ }^{13}$ A minimum of 172 patients (86 patients per group) is required to provide $80 \%$ power with a two-sided $\alpha$ level of 0.05 by Student's t-test on the ratio of BNP change rate between the luseogliflozin and voglibose groups. With $10 \%$ of the patients estimated to withdraw from participation during the study period, the final enrolment target was set at 190 patients (95 patients per group).

\section{Analysis plan}

In the efficacy analysis, the primary population comprises the full analysis set (FAS), defined as all randomised patients who receive one dose of study drug and are followed up at least once. The patients with no BNP data and the patients who withdraw or discontinue treatment will be excluded from the FAS. Missing values at 4, 12 and 24 weeks will be replaced by the last observed value for that variable (last observation carried forward). In the primary outcome analysis, baseline observation carried forward analysis will be also performed. Efficacy analysis will be performed according to the treatment to which the patients are randomly assigned, based on the intention-to-treat analysis. The primary outcome analysis will be based on an analysis of covariance (ANCOVA) $(\alpha=0.05$, level of significance) for the ratio of BNP change rate in the FAS. Adjusted covariates will include the assigned treatment (luseogliflozin, voglibose), baseline age ( $<65$ or $\geq 65$ years), baseline HbA1c $(<8.0$ or $\geq 8.0 \%)$, baseline BNP $(<100$ or $\geq 100 \mathrm{pg} / \mathrm{mL})$, baseline renal function (eGFR $\geq 60$ or $<60 \mathrm{~mL} / \mathrm{min} / 1.73 \mathrm{~m}^{2}$ ), use of thiazolidine or not at baseline and presence or absence of AF and AFL 
at baseline as stratified factors of randomisation. Furthermore, BNP change rate, ratio of BNP change rate and 95\% CIs will be calculated. The same ANCOVA analysis as for the primary outcome will be performed for the ratio of BNP change rate at 4 weeks and 24 weeks between the two groups.

Prespecified subgroup analyses will be performed on the primary outcome using ANCOVA (covariates: assigned treatment and BNP at screening) in the following subgroups: baseline age $(<65$ or $\geq 65$ years), baseline HbA1c $(<8.0$ or $\geq 8.0 \%)$, baseline BNP $(<100$ or $\geq 100 \mathrm{pg} / \mathrm{mL}$ ), baseline renal function (eGFR $\geq 60$ or $<60 \mathrm{~mL} / \mathrm{min} / 1.73 \mathrm{~m}^{2}$ ), use of thiazolidine or not at baseline baseline body weight $(<60 \mathrm{~kg}, \geq 60 \mathrm{~kg})$ and presence or absence of AF and AFL at baseline. Furthermore, exploratory analysis on the primary outcome will be performed in subgroups based on blood pressure, heart rate, waist circumference, cardiovascular risk factors (hypertension, T2DM, hyperuricaemia, family history and smoking), alcohol consumption, regular medication and serum lipid levels (details listed in online supplementary file 3 ).

The key secondary outcomes, difference in $\mathrm{E} / \mathrm{e}^{\prime}$, left ventricular ejection fraction, body weight and $\mathrm{HbAlC}$ at 12 weeks between the luseogliflozin and voglibose groups, will be analysed using the same ANCOVA as for the primary outcome. Subgroup analysis for the key secondary outcomes will be performed in the same subgroups as for the primary outcome analysis. The following secondary outcomes will be also analysed using the same analysis plan: E/e', left ventricular ejection fraction, body weight and $\mathrm{HbAlC}$ at 4 and 24 weeks and exploratory parameters at 4,12 , and 24 weeks.

For the safety analysis, the primary population is the safety analysis set (SAFETY), defined as all the patients who receive at least one dose of study drug. Although the patients who withdraw without receiving study drug will be excluded from SAFETY, other patients who withdraw for any other reason will be included. The safety analysis will be performed according to the treatment administered to the patients in practice, based on the as-treated analysis. Analysis of SAEs (MACE, hypoglycaemia and urinary tract infection) will be performed using the Cochran-Mantel-Haenszel test with stratification factors of age ( $<65$ or $\geq 65$ years), baseline HbAlc $(<8.0$ or $\geq 8.0 \%)$, baseline BNP $(<100$ or $\geq 100 \mathrm{pg} /$ $\mathrm{mL}$ ), baseline renal function (eGFR $\geq 60$ or $<60 \mathrm{~mL}$ / $\min / 1.73 \mathrm{~m}^{2}$ ), use of thiazolidine or not and presence or absence of AF and AFL at screening.

All comparisons are planned, and the analyses will be two-sided with $\mathrm{P}$ values $<0.05$ considered statistically significant. All statistical analyses will be performed using IBM SPSS Statistics 24 (IBM, Armonk, NY) and Stata/SE 15.1 for Mac (StataCorp, College Station, TX). The statistical analysis plan will be developed by the principal investigator and a biostatistician prior to the completion of the patient recruitment and database lock.
Ethics and dissemination

\section{Ethics approval and consent to participate}

This study was approved by the Okayama University Graduate School of Medicine, Dentistry and Pharmaceutical Sciences and the Okayama University Hospital Ethics Committee, as well as the ethics committee of each participating centre. This trial will be conducted in compliance with the Declaration of Helsinki.

\section{Consent for publication}

All participants will provide written informed consent prior to participation.

\section{Dissemination policy}

Findings will be published in peer-reviewed journals and presented at local, national and international meetings and conferences to publicise the research to clinicians and commissioners.

\section{Patient and public involvement}

There is no patient and public involvement in this study.

\section{Study status}

Study enrolment was terminated in September 2018 and data collection was completed by the end of December 2018.

\section{DISCUSSION}

The MUSCAT-HF trial is an ongoing, multi-centre, randomised controlled trial designed to investigate the drug efficacy of luseogliflozin to reduce BNP in T2DM patients with HFpEF. Eligible participants will be randomised to receive luseogliflozin or voglibose in addition to their background medication for 24 weeks. The primary endpoint is the percentage change from baseline in BNP level after 12 weeks of treatment. This trial has the potential to provide novel clinical evidence regarding the treatment of HFpEF in the patients with T2DM.

The EMPA-REG OUTCOME and CANVAS trials showed that the treatment of empagliflozin and canagliflozin, respectively, significantly reduced cardiovascular events in T2DM patients with higher cardiovascular risk. ${ }^{6}{ }^{8}$ Specifically, a $35 \%$ and $33 \%$ relative risk reduction in hospitalisation for heart failure was observed in the EMPA-REG OUTCOME and CANVAS trials, respectively. Although a significant reduction in hospitalisation for heart failure was clearly documented, the proportion of the patients with heart failure and reduced or preserved ejection fraction was not reported precisely in either trials. Therefore, the therapeutic effect of SGLT2 inhibitors specifically in the patients with heart failure has yet to be established. At present, HFpEF prognosis cannot be improved with the use of conventional drugs such as an angiotensin-converting enzyme inhibitor, angiotensin receptor blocker, beta blocker or mineralocorticoid receptor blocker. ${ }^{14-17}$ SGLT2 inhibitors therefore represent a promising strategy for the prevention of HFpEF and improvement of HFpEF outcome by improving left ventricular diastolic function 
in the patients with T2DM. A recent small prospective cohort study in 37 patients showed that canagliflozin improved left ventricular diastolic function within 3 months, although the data in terms of prognosis were limited. ${ }^{18}$ Further, several clinical trials to investigate the effect of SGLT2 inhibitors in cardiovascular clinical hard endpoints in HFpEF patients with T2DM are ongoing (EMPEROR-Preserved; ClinicalTrials.gov Identifier: NCT03057951 and DELIVER; ClinicalTrials.gov Identifier: NCT0361921). Although our study focused on BNP as surrogate endpoint for worsening of heart failure, the results will provide the evidence for the drug efficacy of SGLT2 inhibitor on pathophysiological aspects in those patients.

In summary, emerging evidence suggests that SGLT2 inhibitors exert protective effects against cardiovascular events beyond their glucose-lowering capabilities, although further investigation of the mechanisms underlying these effects is warranted. The MUSCAT-HF trial, the results of which are expected to be published in 2019, will provide novel clinical insights into the treatment of the patients with T2DM and HFpEF.

\section{Author affiliations}

${ }^{1}$ Department of Cardiovascular Medicine, Okayama University Graduate School of Medicine, Dentistry and Pharmaceutical Sciences, Okayama, Japan

${ }^{2}$ Department of Cardiovascular Medicine, Iwakuni Clinical Centre, Iwakuni, Japan

${ }^{3}$ Department of Cardiology, Okayama Medical Center, Okayama, Japan

${ }^{4}$ Department of Cardiology, Okayama Rosai Hospital, Okayama, Japan

${ }^{5}$ Department of Cardiology, Mitoyo General Hospital, Kan-onji, Japan

Contributors KE, TM and $\mathrm{HI}$ contributed to the study design. KE, TM, KN, SS, MM, SN, AT and HI contributed to data interpretation and the drafting of the manuscript. All authors read and approved the final manuscript.

Funding This study is funded by Novartis Pharmaceuticals (Basel, Switzerland).

Competing interests $\mathrm{KE}, \mathrm{SS}, \mathrm{MM}, \mathrm{SN}$ and $\mathrm{AT}$ have no competing interests to declare. TM and KN has received honorarium from Novartis Pharmaceuticals (Basel, Switzerland). HI has received research funding and honorarium from Novartis Pharmaceuticals (Basel, Switzerland).

Patient consent for publication Not required.

Provenance and peer review Not commissioned; externally peer reviewed.

Data sharing statement All data are available upon request.

Open access This is an open access article distributed in accordance with the Creative Commons Attribution Non Commercial (CC BY-NC 4.0) license, which permits others to distribute, remix, adapt, build upon this work non-commercially, and license their derivative works on different terms, provided the original work is properly cited, appropriate credit is given, any changes made indicated, and the use is non-commercial. See: http:// creativecommons.org/licenses/by-nc/4.0/.

\section{REFERENCES}

1. Lindman BR, Dávila-Román VG, Mann DL, et al. Cardiovascular phenotype in HFpEF patients with or without diabetes: a RELAX trial ancillary study. J Am Coll Cardiol 2014;64:541-9.

2. Kannel WB, Hjortland M, Castelli WP. Role of diabetes in congestive heart failure: the Framingham study. Am J Cardiol 1974;34:29-34.

3. Turnbull FM, Abraira C, Anderson RJ, et al. Intensive glucose control and macrovascular outcomes in type 2 diabetes. Diabetologia 2009;52:2288-98.

4. Kim J, Nakatani S, Hashimura K, et al. Abnormal glucose tolerance contributes to the progression of chronic heart failure in patients with dilated cardiomyopathy. Hypertens Res 2006;29:775-82.

5. Chiasson JL, Josse RG, Gomis R, et al. Acarbose treatment and the risk of cardiovascular disease and hypertension in patients with impaired glucose tolerance: the STOP-NIDDM trial. JAMA 2003;290:486-94.

6. Zinman B, Wanner C, Lachin JM, et al. Empagliflozin, cardiovascular outcomes, and mortality in type 2 diabetes. N Engl $J$ Med 2015;373:2117-28.

7. Fitchett D, Zinman B, Wanner C, et al. Heart failure outcomes with empagliflozin in patients with type 2 diabetes at high cardiovascular risk: results of the EMPA-REG OUTCOME $®$ trial. Eur Heart $J$ 2016;37:1526-34.

8. Neal B, Perkovic V, Mahaffey KW, et al. Canagliflozin and cardiovascular and renal events in type 2 diabetes. N Engl J Med 2017;377:644-57.

9. Radholm K, Figtree G, Perkovic V, et al. Canagliflozin and heart failure in type 2 diabetes mellitus: results from the CANVAS Program (Canagliflozin Cardiovascular Assessment Study). Circulation 2018;138:458-68.

10. McMurray JJ, Adamopoulos S, Anker SD, et al. ESC Guidelines for the diagnosis and treatment of acute and chronic heart failure 2012: The Task Force for the Diagnosis and Treatment of Acute and Chronic Heart Failure 2012 of the European Society of Cardiology. Developed in collaboration with the Heart Failure Association (HFA) of the ESC. Eur Heart J 2012;33:1787-847.

11. Rousseau MF, Gurné O, Duprez D, et al. Beneficial neurohormonal profile of spironolactone in severe congestive heart failure: results from the RALES neurohormonal substudy. J Am Coll Cardiol 2002;40:1596-601.

12. Tsutamoto T, Wada A, Maeda K, et al. Effect of spironolactone on plasma brain natriuretic peptide and left ventricular remodeling in patients with congestive heart failure. J Am Coll Cardiol 2001;37:1228-33.

13. Solomon SD, Zile M, Pieske B, et al. The angiotensin receptor neprilysin inhibitor LCZ696 in heart failure with preserved ejection fraction: a phase 2 double-blind randomised controlled trial. Lancet 2012;380:1387-95.

14. Lund LH, Benson L, Dahlström U, et al. Association between use of $\beta$-blockers and outcomes in patients with heart failure and preserved ejection fraction. JAMA 2014;312:2008-18.

15. Massie BM, Carson PE, McMurray JJ, et al. Irbesartan in patients with heart failure and preserved ejection fraction. N Engl J Med 2008;359:2456-67.

16. Solomon SD, Claggett B, Lewis EF, et al. Influence of ejection fraction on outcomes and efficacy of spironolactone in patients with heart failure with preserved ejection fraction. Eur Heart $J$ 2016:37:455-62.

17. Holland DJ, Kumbhani DJ, Ahmed SH, et al. Effects of treatment on exercise tolerance, cardiac function, and mortality in heart failure with preserved ejection fraction. A meta-analysis. J Am Coll Cardiol 2011;57:1676-86.

18. Matsutani D, Sakamoto M, Kayama $Y$, et al. Effect of canagliflozin on left ventricular diastolic function in patients with type 2 diabetes. Cardiovasc Diabetol 2018;17:73. 
Correction: The effect of luseogliflozin and alpha-glucosidase inhibitor on heart failure with preserved ejection fraction in diabetic patients: rationale and design of the MUSCAT-HF randomised controlled trial

Ejiri K, Miyoshi T, Nakamura K, et al. The effect of luseogliflozin and alpha-glucosidase inhibitor on heart failure with preserved ejection fraction in diabetic patients: rationale and design of the MUSCAT-HF randomised controlled trial. BMJ Open 2019;9:e026590. doi: 10.1136/bmjopen-2018-026590.

This article was previously published with an error.

Under 'Finding' section, the authors have stated, "This study is funded by Novartis Pharmaceuticals (Basel, Switzerland)."

The correct sentence is as follows:

"This study is funded by Novartis Pharma K. K."

Open access This is an open access article distributed in accordance with the Creative Commons Attribution Non Commercial (CC BY-NC 4.0) license, which permits others to distribute, remix, adapt, build upon this work non-commercially, and license their derivative works on different terms, provided the original work is properly cited, appropriate credit is given, any changes made indicated, and the use is non-commercial. See: http://creativecommons.org/licenses/by-nc/4.0/.

(c) Author(s) (or their employer(s)) 2019. Re-use permitted under CC BY-NC. No commercial re-use. See rights and permissions. Published by BMJ.

BMJ Open 2019;9:e026590corr1. doi:10.1136/bmjopen-2018-026590corr1

A) Check for updates 